\title{
Physical frailty and decline in general and specific cognitive abilities
}

\section{Marcus Richards}

Frailty has been conceptualised as reduction in the functional capacity of multiple physiological systems, and results in a wide range of outcomes, including physical limitations, impaired activities of daily living, falls and fractures, hospitalisation and mortality. ${ }^{1}$ These outcomes also include cognitive decline, ${ }^{1}$ which in combination with psychiatric morbidity is the biggest cause of disability in the UK and other Western countries.

Gale et al report that frailty predicts decline in a range of cognitive functions in the Lothian Birth Cohort 1936. Frailty predicted those of fluid function (memory, processing speed and visuospatial) and crystallised ability, as measured by pronunciation of irregular words. ${ }^{2}$ Frailty was defined by the Fried phenotype, that is, the presence of at least three of: weakness (measured by grip strength); self-reported exhaustion; slow gait speed; unintentional weight loss (estimated from low body mass index) and low physical activity. In addition to age, sex and education, associations were independent of two important sources of potential confounding: a range of chronic physical conditions, suggesting that frailty is not merely marking general morbidity burden; and depressive symptoms, further suggesting that exhaustion is not a proxy for this well-known dementia risk factor. Finally, results were essentially unchanged when those scoring below the standard Mini-Mental Status Examination clinical threshold were excluded, indicating that associations with frailty operate within the framework of normal cognitive ageing.

So what is it about 'frailty' here that matters? Such indices are generally intended to be more than the sum of their parts, an approach that can be contentious (as it is with the 'metabolic syndrome'), but has long seemed promising for dementia prediction. ${ }^{3}{ }^{4}$ An assumption of both those latter studies is that individual factors weakly predict outcomes, if at all, but better discriminate risk in combination. For example, Song et $a l^{4}$ compiled a list of 23 'non-traditional'

MRC Unit for Lifelong Health and Ageing, UCL, London, UK

Correspondence to Professor Marcus Richards, MRC Unit for Lifelong Health and Ageing at UCL, UCL, London WC1B 5JU, UK; m.richards@ucl.ac.uk risk factors for Alzheimer's disease (AD), few of which individually predicted this outcome, and some barely seemed relevant at all (eg, trouble with skin, feet or ankles). Yet their aggregate, which the authors refer to as representing 'general health', more reliably predicted dementia and death. The Fried phenotype does not behave quite like this. As noted, in the study by Gale et al, this predicted cognitive function independently of other key health indicators; second, most if not all of the components themselves individually predict cognitive function. On face value it would therefore have been surprising had the accumulation of these factors not predicted this outcome. However, a closer look reveals a more complex story. Of the five Fried phenotype components, physical activity and gait speed are the best known associates of cognition, with strong mechanistic plausibility (eg, neurogenesis, neuroanatomical common cause, reduction of cardiovascular risk). Still on motor function, weakness (grip strength) is also associated with cognition, including in the Lothian 1936 and 1921 cohorts. $^{56}$ The criterion of unintentional weight loss is less certain, and belongs to a small group of dementia risk factors that seem to invert their direction with age: for example, obesity and hypertension in midlife but weight loss and hypotension in later life. However, while there are debates about the interpretation of this inversion, most would regard cachexia in old age as a clinical sign. Exhaustion is probably the least understood of the Fried components, although a recent review found that its more extreme manifestation, exhaustion disorder (ED; 'burnout'), is associated with impaired executive function, attention and memory. ${ }^{7}$ The association between frailty and cognition was independent of depressive symptoms in the study by Gale et al, as mentioned above; consistent with this, while there is some overlap between ED and these symptoms, ED lacks the features of low mood, anhedonia, self-blame and suicidal ideation that are hallmarks of major depressive disorder. ${ }^{7}$ Interestingly, one study in this review reported decreased volumes of structures within the basal ganglia, which are involved in movement control and thus may share common cause with some motor aspects of the Fried phenotype. ${ }^{8}$
Gale et al suggest several priorities for further research. One should be to test 'lead-lag' effects, that is, whether changes in cognition follow changes in frailty, or vice versa, or whether there is interplay between these. While it is worth noting that there was little evidence for shared declines in physical and cognitive functions in the Lothian cohorts, ${ }^{56}$ multilevel growth curve analysis using the HonoluluAsia Aging Study suggests that withinperson increases in frailty predicted cognitive decline. ${ }^{9}$ This does not rule out dynamic interplay however, since Gale et al also found that decline in cognitive function predicted Fried frailty in the Lothian Birth Cohort 1936 (ref. 29 in target article), as did childhood cognition. ${ }^{10}$ It would therefore be interesting to see how much of the frailty-cognition associations in the present article are explained by the latter; and what a crosslagged model would look like that incorporates cognitive decline prior to the frailty measure. Another research priority suggested by Gale et al is to investigate the role of neuropathology in associations between frailty and cognition. So far, this has been addressed the other way round using the Rush Memory and Aging Project. People with a high frailty score had a weakened direct association between neuritic plaques, diffuse plaques and neurofibrillary tangles, and a clinical diagnosis of $\mathrm{AD},{ }^{11}$ thus $\mathrm{AD}$ pathology may remain clinically silent for longer in people with low frailty. The index of frailty used in that study was a composite of 41 items, many of which are markers of general health, so it would be intriguing to see if there is similar modification by the Fried phenotype, with the kind of controls used by Gale et al.

Frailty is potentially reversible. ${ }^{12}$ An important implication of the study by Gale et al, therefore, is that frailty should be added to our list of known modifiable risk factors for cognitive impairment. What might the practical consequences of this be? The UK's National Institute for Health and Care Excellence and National Health Service general practice contract for 2017/2018, the Canadian Frailty Network and the Asia-Pacific Clinical Practice Guidelines for the Management of Frailty all recommend identification of frailty in older people, especially those with multimorbidity. Most of these guidelines are linked to strategies for clinical management of frailty, for example, the Comprehensive Geriatric Assessment. However, much research is still required before recommendations can be formalised for advancing evidence-based 
treatment options, and for cost-effective care delivery strategies. ${ }^{12}$ As with dementia, routing screening of the general older population for frailty is controversial, although at least there are comprehensive clinical indications for frailty. The study by Gale et al can only help draw our attention to these vital issues.

Funding The authors have not declared a specific grant for this research from any funding agency in the public, commercial or not-for-profit sectors.

Competing interests None declared.

Patient consent for publication Not required.

Provenance and peer review Commissioned; internally peer reviewed.

(c) Author(s) (or their employer(s)) 2020. No commercial re-use. See rights and permissions. Published by BMJ.

\section{A) Check for updates}

To cite Richards M. J Epidemiol Community Health 2020;74:105-106.

Received 21 October 2019

Accepted 21 October 2019

Published Online First 5 November 2019

\section{CLinked}

http://dx.doi.org/10.1136/jech-2019-213280

J Epidemiol Community Health 2020;74:105-106. doi:10.1136/jech-2019-213410

\section{REFERENCES}

1 Vermeiren S, Vella-Azzopardi R, Beckwée D, et al. Gerontopole Brussels Study Group. frailty and the prediction of negative health outcomes: a metaanalysis. J Am Med Dir Assoc 2016;17:1163.e1-1163. e17.

2 Gale C, Ritchie S, Starr J, et al. Physical frailty and decline in general and specific cognitive abilities: the Lothian birth cohort 1936. J Epidemiol Community Health 2020;74:108-13.

3 Stephan BCM, Kurth T, Matthews FE, et al. Dementia risk prediction in the population: are screening models accurate? Nat Rev Neurol 2010;6:318-26.

4 Song X, Mitnitski A, Rockwood K. Nontraditional risk factors combine to predict Alzheimer disease and dementia. Neurology 2011;77:227-34.

5 Deary IJ, Johnson W, Gow AJ, et al. Losing one's grip: a bivariate growth curve model of grip strength and nonverbal Reasoning from age 79 to 87 years in the Lothian birth cohort 1921. J Gerontol B Psychol Sci Soc Sci 2011;66:699-707.
6 Ritchie SJ, Tucker-Drob EM, Starr JM, et al. Do cognitive and physical functions age in concert from age 70 to 76 ? Evidence from the Lothian birth cohort 1936. Span J Psychol 2016;19:12.

7 Grossi G, Perski A, Osika W, et al. Stress-related exhaustion disorder - clinical manifestation of burnout? A review of assessment methods, sleep impairments, cognitive disturbances, and neurobiological and physiological changes in clinical burnout. Scand J Psychol 2015;56:626-36.

8 Blix E, Perski A, Berglund $H$, et al. Long-Term occupational stress is associated with regional reductions in brain tissue volumes. PLoS One 2013;8:e64065.

9 Armstrong JJ, Godin J, Launer LJ, et al. Changes in Frailty Predict Changes in Cognition in Older Men: The Honolulu-Asia Aging Study. JAD 2016;53:1003-13.

10 Gale CR, Booth T, Starr JM, et al. Intelligence and socioeconomic position in childhood in relation to frailty and cumulative allostatic load in later life: the Lothian birth cohort 1936. J Epidemiol Community Health 2016;70:576-82.

11 Wallace LMK, Theou O, Godin J, et al. Investigation of frailty as a moderator of the relationship between neuropathology and dementia in Alzheimer's disease: a cross-sectional analysis of data from the rush memory and aging project. Lancet Neurol 2019;18:177-84.

12 Dent E, Martin FC, Bergman H, et al. Management of frailty: opportunities, challenges, and future directions. The Lancet 2019;394:1376-86. 